\title{
Risk factors and outcomes of post- traumatic endophthalmitis: a retrospective single-center study
}

\author{
Nawat Watanachai', Janejit Choovuthayakorn ${ }^{1 *}$ DD, Susama Chokesuwattanaskul', Chaipot Photcharapongsakul', \\ Praelada Wongsirimeteekul ${ }^{1}$, Phichayut Phinyo ${ }^{2,3,4}$, Voraporn Chaikitmongkol', Paradee Kunavisarut ${ }^{1}$, \\ Pongsant Supreeyathitikul ${ }^{1}$ and Direk Patikulsila ${ }^{1}$
}

\begin{abstract}
Background: To describe the epidemiology, characteristics, risk factors, and outcomes of post-traumatic endophthalmitis.
\end{abstract}

Main body: Medical records of consecutive open globe injury patients admitted and primarily treated between January 2006 and December 2016 were retrospectively reviewed. Patients were defined as having or not having associated endophthalmitis. Data of demographics, injury characteristics, clinical presentations, and visual outcomes were collected. The potential risks and significant factors for visual outcomes of post-traumatic endophthalmitis were determined. There were 591 patients included in this study. Among these, 118 patients were clinically diagnosed as having accompanied endophthalmitis. Higher proportions of intraocular foreign body (IOFB) (55.1\% vs. $27.3 \%)$ and injury related to high-velocity objects (55.9\% vs. $32.6 \%)$ were noted in patients with endophthalmitis compared to patients without endophthalmitis. Anterior wound location (odds ratio [OR], 2.0; 95\% confidence interval $[\mathrm{Cl}], 1.1$ to $3.7 ; P=0.020)$, presence of IOFB $(\mathrm{OR}, 1.9 ; 95 \% \mathrm{Cl} 1.2$ to $3.0 ; P=0.005)$, and delayed presentation of $>24 \mathrm{~h}(\mathrm{OR}, 3.9 ; 95 \% \mathrm{Cl} 2.3$ to $6.4 ; P<0.001)$ were significant risk factors for associated endophthalmitis. Final visual acuity (VA) of the overall population improved significantly from $2.4(0.6) \log M A R$ to $1.4(0.1) \log M A R, P<0.001$, however, patients in the endophthalmitis group achieved a worse final VA than the non-endophthalmitis group (66.1\% vs. $43.5 \%, P<0.001)$.

Conclusion: High proportions of post-traumatic endophthalmitis patients had subsequent poor visual outcomes. Therefore, safety and protective measurements, especially when performing activities related to high-velocity objects, and the institution of prophylactic antibiotics in high-risk groups should be promptly considered to reduce the incidence.

\section{Introduction}

Ocular trauma, particularly open globe injury (OGI), is one of the major causes of acquired visual loss of the general population at all ages. Visual disability after ocular trauma can be determined by several factors, including mechanism of injury, the severity of damaged ocular tissue, and associated complications [1-7]. One of the

\footnotetext{
*Correspondence: janejit.c@cmu.ac.th

'Department of Ophthalmology, Faculty of Medicine, Chiang Mai University, 110 Intavaroros Road, Maung, Chiang Mai 50200, Thailand

Full list of author information is available at the end of the article
}

most deleterious complications is post-traumatic endophthalmitis, which is caused by inoculation of pathogens, either normal ocular flora or other environmental microorganisms, through a breakdown of a corneoscleral shell into an eyeball. Commonly, the condition results in profound visual loss following OGI. Compared to other intraocular infections (such as post-operative endophthalmitis), post-traumatic endophthalmitis patients had worse final visual outcomes [8-10]. Therefore, a close monitoring of cases with high risk for endophthalmitis, early recognition, and prompt management are crucial 
steps to decrease the incidence and improve the visual outcomes among these patients.

This study aimed to describe the etiology, clinical characteristics, and outcomes of patients with posttraumatic endophthalmitis treated at a tertiary center in Northern Thailand. Information may provide additional insights for clinicians regarding prevention, management, and visual prognosis of the condition.

\section{Methods}

This observational study was conducted in accordance with the tenets of the Declaration of Helsinki. The protocol was approved by the University Research Ethics Committee. Due to anonymized data, informed consent was exempted. Patients with OGI, defined as a fullthickness laceration of either cornea or sclera according to The Birmingham Eye Trauma Terminology System (BETT), admitted at Chiang Mai University hospital between January 2006 and December 2016 were identified [11]. Medical records of consecutive patients who admitted for primary ophthalmic management at this hospital and followed for at least 1 month were reviewed. In a protocol-specific record form, demographics including age, gender, activity and mechanism of injury, and time interval from injury to ophthalmic evaluation were collected. Besides, details of globe injury including laterality, initial visual acuity (VA), anterior and posterior segment abnormalities, and imaging investigations (B-scan ultrasonography, skull film, or computerized tomography) were assessed. Wound location was defined as zone I (laceration limited to the cornea), zone II (laceration involving the sclera, within $5-\mathrm{mm}$ of the corneoscleral limbus), and zone III (laceration involving posterior sclera, beyond 5-mm of the corneoscleral limbus) [12]. Presence of endophthalmitis was determined by treating retinal physicians (DP, JC, NW, PK, and VC) using the following clinical symptoms and signs: eye pain and redness; deteriorating in VA; purulent discharge; chemosis; eyelid edema; fibrin and/or hypopyon in the anterior chamber; and vitreous opacification.

For medical management, all OGI patients immediately received empirical intravenous vancomycin $1 \mathrm{~g}$ every $12 \mathrm{~h}$ and ceftazidime $1 \mathrm{~g}$ every $8 \mathrm{~h}$ (starting at presentation to this hospital or before if referred from the outside centers) and continued for 3 to 5 days. For patients who presented and/or developed symptoms and signs of endophthalmitis as well as patients who presented with high risk of infection (delayed presentation and having intraocular foreign body, IOFB), intravitreal injections of vancomycin $1 \mathrm{mg}$ in $0.1 \mathrm{ml}$ and ceftazidime $2.25 \mathrm{mg}$ in $0.1 \mathrm{ml}$ were administered. Additionally, in patients with evidence of fungus infection from the intraocular fluid investigation, an intravitreal amphotericin B $(5 \mu \mathrm{g}$ in $0.1 \mathrm{ml})$ was injected. The application of intensive topical antibiotics (cefazolin $33 \mathrm{mg} / \mathrm{ml}$ and gentamicin $14 \mathrm{mg} / \mathrm{ml}$ ), $1 \%$ prednisolone acetate, and an oral systemic antibiotic (ciprofloxacin $500 \mathrm{mg}$ twice a day) were considered by treating physicians based on the severity of the infection and associated ocular injuries. Any surgical management, including pars plana vitrectomy (PPV), was reviewed. In endophthalmitis patients, microbiological results from both aqueous and vitreous fluid samplings (which were examined and cultured on conventional media including blood agar, chocolate agar, thioglycolate broth, and Sabouraud dextrose agar) were collected. Final anatomical and visual outcomes at the last follow-up visit were recorded.

\section{Statistical analysis}

Categorical data were described as frequency and percentage, while continuous data as mean (standard deviation, SD) or median (interquartile range, IQR). Snellen VA was calculated into a logarithm of the minimum angle of resolution (logMAR) unit for the statistical analysis. Patients were categorized into OGI with accompanied intraocular infection (endophthalmitis group) and OGI without the intraocular infection (nonendophthalmitis group). In comparative statistical analysis, categorical data were assessed by Fisher's exact or Chi-squared test, while independent T-test or nonparametric test were used for continuous variables. Risk of endophthalmitis (dependent variable) was estimated by multivariable logistic regression. Independent variables included in a model were factors that showed significant differences in univariable calculation. In addition, prognostic factors associated with poor visual outcome (defined as final VA of less than 20/400) among endophthalmitis patients were also evaluated in a model that included age, gender, initial VA, wound characteristics (location, self-sealing, and contamination), presence of relative afferent pupillary defect (RAPD), retinal detachment, presence of IOFB, vitreous and uveal tissue prolapse, positive culture results, performing PPV, and time from injury to hospital as independent factors. Data were analyzed by the SPSS version 16.0 software (SPSS Inc, Chicago, IL, USA). A $P$ value of less than 0.05 was considered a statistical significance.

\section{Results}

A total of 591 OGI patients (591 eyes) with a median (IQR) follow-up of 5.5 (2 to 17) months were included in this study. Three hundred and seventy-two patients (62.9\%) were referred from outside centers. Of all patients, 118 were clinically diagnosed with endophthalmitis. The demographics and injury characteristics of overall OGI patients categorized by accompanying endophthalmitis are summarized in Table 1. Overall, there were no differences in mean age $(P=0.655)$, gender $(P=0.747)$, and 
Table 1 Demographics and injury characteristics of open globe injury patients divided into endophthalmitis and nonendophthalmitis groups

\begin{tabular}{|c|c|c|c|c|}
\hline Characteristics & Total $(N=591)$ & $\begin{array}{l}\text { Endophthalmitis } \\
\text { group }(N=118)\end{array}$ & $\begin{array}{l}\text { Non-endophthalmitis } \\
\text { group }(N=473)\end{array}$ & $P$ value \\
\hline Mean age (SD), year & $39.0(18.2)$ & $39.2(17.2)$ & $38.9(18.4)$ & 0.655 \\
\hline Gender (male/female) & $524 / 67$ & 106/12 & $418 / 55$ & 0.747 \\
\hline Laterality (right/left) & $298 / 293$ & $54 / 64$ & $244 / 229$ & 0.303 \\
\hline Age group (year), n (\%) & & & & 0.246 \\
\hline 0 to 20 & $105(17.8)$ & $18(15.3)$ & $87(18.4)$ & \\
\hline$>20$ to 40 & $200(33.8)$ & $39(33.1)$ & $161(34.0)$ & \\
\hline$>40$ to 60 & $225(38.1)$ & $53(44.9)$ & $172(36.4)$ & \\
\hline$>60$ & $61(10.3)$ & $8(6.8)$ & $53(11.2)$ & \\
\hline Mechanism of injury, n (\%) & & & & $<0.001$ \\
\hline IOFB & $194(32.8)$ & $65(55.1)$ & $129(27.3)$ & \\
\hline Penetration/perforation & 287 (18.6) & $49(41.5)$ & $238(50.3)$ & \\
\hline Rupture & $110(48.6)$ & $4(3.4)$ & $106(22.4)$ & \\
\hline Injury site, n (\%) & & & & $<0.001$ \\
\hline Workplace based & $375(63.5)$ & $96(81.4)$ & $279(59.0)$ & \\
\hline Outdoor based & $181(30.6)$ & $18(15.3)$ & $163(34.5)$ & \\
\hline Home and indoor based & $35(5.9)$ & $4(3.4)$ & $31(6.6)$ & \\
\hline Object causing injury, n (\%) & & & & $<0.001$ \\
\hline Mowing related projectile objects & $147(24.9)$ & $53(44.9)$ & $94(19.9)$ & \\
\hline Chiseling/hammering related projectile objects & $73(12.4)$ & $13(11.0)$ & $60(12.7)$ & \\
\hline Other metallic objects & $59(10.0)$ & 16 (13.6) & $43(9.1)$ & \\
\hline Sticky wood/wooden object & $90(15.2)$ & $15(12.7)$ & $75(15.9)$ & \\
\hline Explosive object & $54(9.1)$ & $7(5.9)$ & $47(9.9)$ & \\
\hline Hit by other blunt objects & $78(13.2)$ & $5(4.2)$ & $73(15.4)$ & \\
\hline Needle/knife & $20(3.4)$ & $4(3.4)$ & $16(3.4)$ & \\
\hline Glass & $55(9.3)$ & $2(1.7)$ & $53(11.2)$ & \\
\hline Unknown & $15(2.5)$ & $3(2.5)$ & $12(2.5)$ & \\
\hline Address of injury, n (\%) & & & & $<0.001$ \\
\hline Rural & $359(60.7)$ & $90(76.3)$ & $269(56.9)$ & \\
\hline Urban & $232(39.7)$ & $28(23.7)$ & $204(43.1)$ & \\
\hline Soil/vegetation contaminated injury, n (\%) & $237(40.1)$ & 68 (57.6) & 169 (37.5) & $<0.001$ \\
\hline
\end{tabular}

IOFB intraocular foreign body, SD standard deviation

laterality $(P=0.303)$ between patients with and without endophthalmitis. However, patients in the endophthalmitis group tended to have sustained injuries related to IOFB $(55.1 \%$ vs. $27.3 \%)$ and high-velocity objects $(55.9 \%$ vs. $32.6 \%)$, and lived in rural areas (76.3\% vs. $56.9 \%)$ compared to patients in the non-endophthalmitis group.

\section{Clinical characteristics}

For a mean (SD) presenting VA, patients in the endophthalmitis group had poorer vision than patients without endophthalmitis [2.3 (0.7) logMAR unit vs. 2.0 (0.9) logMAR unit, $P<0.001$ ). Wound characteristics (location, contamination, and self-sealing], and vitreous and uveal tissue prolapse were significantly different between the two groups (all $P$ values $<0.001$ ). Considering the time interval from injury to the hospital, a median (IQR) of 24 (7 to 90) hours was observed for the overall cases. However, the proportions of patients with delayed presentation $>24 \mathrm{~h}$ and the patients who received primary surgery $>24 \mathrm{~h}$ were significantly higher in the endophthalmitis group, compared to the non-endophthalmitis group $(78.8 \%$ vs. $40.4 \%, P<0.001$ and $94.9 \%$ vs. $65.0 \%$, $P<0.001$, respectively). Among 284 patients who had a delayed presentation (with subsequent delayed surgical interventions), the attributable factors included a lack of awareness to visit a healthcare professional after eye 
injury (209, 73.6\%), difficulty in travelling and accessing the healthcare facility $(45,15.8 \%)$, a delayed diagnosis (19, 6.7\%), and undetermined in 11 (3.4\%) patients. Table 2 demonstrates the detailed clinical manifestations of OGI patients in the endophthalmitis and nonendophthalmitis groups.

\section{Risks factors for endophthalmitis}

In multivariable analysis, factors associated with development of endophthalmitis following OGI were Zone I wound location (odds ratio [OR], 2.0; 95\% confidence interval $[\mathrm{CI}], 1.1$ to $3.7 ; P=0.020$ ), presence of IOFB (OR, 1.9; $95 \%$ CI 1.2 to $3.0 ; P=0.005$ ), and delayed presentation $>24 \mathrm{~h}(\mathrm{OR}, 3.9 ; 95 \% \mathrm{CI} 2.3$ to $6.4 ; P<0.001)$ (Table 3). The probabilities of endophthalmitis in patients with these factors are shown in Table 4.

\section{Microbiological proven}

For 118 endophthalmitis patients, aqueous and vitreous fluid collected at presentation and/or at beginning of the operations were subjected for microbiological analysis (but not for fluid obtained from a vitrectomy cassette). Microbiological etiologies were confirmed in 25 (21.2\%) patients ( 2 had positive results from aqueous and vitreous cultures and 23 from vitreous cultures only). Endophthalmitis patients with positive cultures had a lesser proportion of a self-sealing wound ( $24 \%$ vs. $49.5 \%, P=0.040)$, a higher proportion of enucleation/evisceration $(40 \%$ vs. $15.1 \%, P=0.013$, and worse final VA ( $88 \%$ vs. $60.2 \%)$ compared to negative culture patients (Table 5). Table 6 describes the microbiological results of endophthalmitis patients with positive cultures. The most common microbiological spectrum identified was gram-positive organism (including Bacillus spp. and coagulase-negative Staphylococcus). Mixed organisms were cultured in 3 cases and fungus in 1 patient.

\section{Treatments and outcomes}

Intravenous antibiotics treatments were given prior to the presentation to our hospital in 372 referral patients (96 diagnosed as endophthalmitis and 276 nonendophthalmitis) and at the time of presentation in 219 patients who were primarily managed at this hospital. No one received the intravitreal antibiotic injections before the presentation. However, for 118 OGI patients with endophthalmitis and 90 OGI patients with highrisk characteristics for infection (delayed presentation and retained IOFB), the injections were performed at the presentation to this hospital. Of 591 OGI patients, 454 (76.8\%) required primary wound repair, whereas 137 (23.2\%) had a self-sealing wound. Characteristics of patients with self-sealing wounds are described in Table 7. Of note, a higher distribution of zone I injury, presence of IOFB, endophthalmitis, and delayed presentation to the hospital were observed in patients with a self-sealing wound compared to patients requiring primary wound repair ( $P$ values $<0.001)$. In this study, none of the patients without presenting endophthalmitis developed the infection during treatments and follow-up.

For overall OGI, the proportions of patients who underwent PPV or enucleation/evisceration were higher in the endophthalmitis compared to the non-endophthalmitis

Table 2 Clinical presentations of open globe injury patients by endophthalmitis groups

\begin{tabular}{|c|c|c|c|c|}
\hline Clinical characteristics & Total $(N=591)$ & $\begin{array}{l}\text { Endophthalmitis } \\
\text { group }(N=118)\end{array}$ & $\begin{array}{l}\text { Non-endophthalmitis } \\
\text { group }(N=473)\end{array}$ & $P$ value \\
\hline Wound location, n (\%) & & & & $<0.001$ \\
\hline Zone I & $281(47.5)$ & $80(67.8)$ & $201(42.5)$ & \\
\hline Zone II & $166(28.1)$ & $21(17.8)$ & $145(30.7)$ & \\
\hline Zone III & $144(24.4)$ & $17(14.4)$ & $127(26.8)$ & \\
\hline Lens capsule rupture, n (\%) & $104(17.6)$ & $24(20.3)$ & $80(16.9)$ & 0.422 \\
\hline Vitreous prolapsed, n (\%) & $138(23.4)$ & $10(8.5)$ & $128(27.1)$ & $<0.001$ \\
\hline Uveal tissue prolapsed, n (\%) & $261(44.2)$ & $34(28.8)$ & $227(48.0)$ & $<0.001$ \\
\hline Retinal detachment, n (\%) & $133(22.5)$ & $26(22.0)$ & $107(22.6)$ & 0.904 \\
\hline Choroidal detachment, n (\%) & $72(12.2)$ & $8(6.8)$ & $64(13.5)$ & 0.063 \\
\hline RAPD, n (\%) & $184(31.1)$ & $36(30.5)$ & $148(31.3)$ & 0.912 \\
\hline Presenting to hospital > 24 h, n (\%) & $284(48.1)$ & $93(78.8)$ & $191(40.4)$ & $<0.001$ \\
\hline Median duration of admission (IQR), day & 9 (6 to 12$)$ & 10 (7 to 14$)$ & $8(6$ to 12$)$ & $<0.001$ \\
\hline Mean final (SD) VA, logMAR ${ }^{a}$ & $1.4(1.1)$ & $1.9(1.0)$ & $1.3(1.1)$ & $<0.001$ \\
\hline Final VA worsen than 20/400, n (\%) ${ }^{a}$ & $282(48.0)$ & $78(66.1)$ & $204(43.5)$ & $<0.001$ \\
\hline
\end{tabular}

$R A P D$ relative afferent pupillary defect, $I Q R$ interquartile range, $V A$ visual acuity, $S D$ standard deviation, logMAR logarithm of the minimum angle of resolution, $F V A$ $V A$ at final follow-up visit

${ }^{\mathrm{a}} \mathrm{VA}$ at final follow-up could not be determined in 4 patients of non-endophthalmitis group 
Table 3 Multivariable regression for risk of endophthalmitis development following open globe injury by full analysis and reduced model analysis

\begin{tabular}{|c|c|c|c|c|c|c|}
\hline \multirow[t]{2}{*}{ Variables } & \multicolumn{3}{|l|}{ Full analysis } & \multicolumn{3}{|c|}{ Backward LR } \\
\hline & Odds ratio & $95 \% \mathrm{Cl}$ & $P$ value & Odds ratio & $95 \% \mathrm{Cl}$ & $P$ value \\
\hline \multicolumn{7}{|l|}{ Wound location } \\
\hline Zone III & - & - & - & - & - & - \\
\hline Zone II & 1.3 & $0.6-2.6$ & 0.521 & 1.1 & $0.6-2.3$ & 0.701 \\
\hline Zone I & 1.9 & $1.1-3.5$ & 0.045 & 2.0 & $1.1-3.7$ & 0.020 \\
\hline Vitreous prolapse & 1.8 & $0.9-3.9$ & 0.124 & NA & & \\
\hline Uveal tissue prolapse & 0.8 & $0.5-1.4$ & 0.510 & NA & & \\
\hline Presence of IOFB & 1.6 & $0.9-2.6$ & 0.078 & 1.9 & $1.2-3.0$ & 0.005 \\
\hline Self-sealed wound & 1.6 & $0.9-2.7$ & 0.107 & NA & & \\
\hline Contaminated wound & 1.3 & $0.8-2.1$ & 0.248 & NA & & \\
\hline Rural address & 0.9 & $0.6-1.7$ & 0.984 & NA & & \\
\hline Time to hospital > $24 \mathrm{~h}$ & 3.3 & $1.8-5.9$ & $<0.001$ & 3.9 & $2.3-6.4$ & $<0.001$ \\
\hline
\end{tabular}

Cl confidence interval, IOFB intraocular foreign body

group [110 (93.2\%) vs. 230 (48.6\%), $P<0.001$ and 24 (20.3\%) vs. 59 (12.5\%), $P=0.04$, respectively]. Among 24 endophthalmitis patients who underwent enucleation/ evisceration, 10 had positive culture results [ 6 out of all 7 patients with Bacillus species infection, 2 of all 3 patients with gram negative organism (Serratia marcescens and Escherichia coli) infection, and 2 of all 3 patients with mixed-organism infection]. A mean (SD) final VA of the overall OGI population significantly improved from 2.4 (0.6) $\log$ MAR to $1.4(0.1) \log$ MAR, $P<0.001$. However, patients in the endophthalmitis group achieved a worse final VA than in the non-endophthalmitis group $(P<$ 0.001). For endophthalmitis patients, presence of RAPD (OR, 9.5; 95\% CI 3.1 to $61.4 ; P=0.024$ ), and positive microbiological culture (OR, 4.7; $95 \%$ CI 1.2 to $21.5 ; P=$ 0.044 ) were indicated factors for poor final visual outcome in the multivariable analysis. Notably, all three endophthalmitis patients with mixed organisms had a final VA worse than 20/400. During the follow-up, 14 patients in the endophthalmitis group developed hypotony compared to 29 patients in the non-endophthalmitis group (11.9\% vs. $6.1 \%, P=0.014), 13$ vs. 41 patients for retinal detachment $(11.0 \%$ vs. $8.7 \%, P=0.181)$, and 8 vs. 34 patients for secondary glaucoma (6.8\% vs. $7.2 \%, P=0.892)$.

\section{Discussion}

In this study, a risk of post-traumatic endophthalmitis among OGI patients is associated with anterior wound location, presence of IOFB, and delay in primary wound closure. Even though vision significantly improved after OGI treatments, an unfavorable visual prognosis was more frequently observed in the endophthalmitis group than in the non-endophthalmitis group.

Infectious endophthalmitis is a severe ophthalmic condition developed from several etiologies such as ocular trauma, previous intraocular surgery, hematologic spreading, and associated corneal ulceration. Therefore, different etiologies contained different incidences, characteristics, and visual outcomes [13-15]. For post-traumatic endophthalmitis, occurrence and visual prognosis are associated with the nature and extent of the injury, the timing of diagnosis and management, and virulence of

Table 4 Probability of endophthalmitis development following open globe injury by associated risk characteristics

\begin{tabular}{|c|c|c|c|c|c|c|c|c|c|}
\hline \multirow{2}{*}{ Wound location } & \multicolumn{9}{|c|}{ Probability of endophthalmitis (\%) } \\
\hline & \multicolumn{6}{|c|}{2 factors } & \multicolumn{3}{|c|}{3 factors } \\
\hline Zone 3 & $x$ & & & $x$ & & & $x$ & & \\
\hline Zone 2 & & $x$ & & & $x$ & & & $x$ & \\
\hline Zone 1 & & & $x$ & & & $x$ & & & $x$ \\
\hline Presence of IOFB & $x$ & $x$ & $x$ & & & & $x$ & $x$ & $x$ \\
\hline \multirow[t]{2}{*}{ Time to hospital > $24 \mathrm{~h}$} & & & & $x$ & $x$ & $x$ & $x$ & $x$ & $x$ \\
\hline & 9.5 & 10.8 & 17.6 & 17.5 & 19.6 & 30.1 & 28.9 & 31.8 & 45.2 \\
\hline
\end{tabular}

Receiver operating curve $(\mathrm{ROC})=0.79$

IOFB intraocular foreign body 
Table 5 Characteristics of endophthalmitis patients by culture results ( $N=118$ )

\begin{tabular}{|c|c|c|c|}
\hline \multirow[t]{2}{*}{ Characteristics } & \multicolumn{2}{|l|}{ Endophthalmitis $(N=118)$} & \multirow[t]{2}{*}{$P$ value } \\
\hline & Positive culture $(N=25)$ & Negative culture $(N=93)$ & \\
\hline Presence of IOFB, n (\%) & $15(60.0)$ & $50(53.8)$ & 0.741 \\
\hline Lens capsule rupture, n (\%) & $8(32.0)$ & $16(17.2)$ & 0.176 \\
\hline Presenting to hospital > 24 h, n (\%) & $17(68.0)$ & $76(81.7)$ & 0.224 \\
\hline Self-sealing wound, n (\%) & $6(24.0)$ & $46(49.5)$ & 0.040 \\
\hline Mean initial (SD) VA, logMAR & $2.4(0.7)$ & $2.3(0.7)$ & 0.382 \\
\hline Receiving intravenous antibiotics prior presentation, n (\%) & $19(76.0)$ & $77(82.8)$ & 0.627 \\
\hline Final VA worsen than $20 / 400, n(\%)^{a}$ & $22(88.0)$ & $56(60.2)$ & 0.018 \\
\hline Enucleation/evisceration, n (\%) & $10(40.0)$ & 14 (15.1) & 0.013 \\
\hline
\end{tabular}

$V A$ visual acuity, $S D$ standard deviation, logMAR logarithm of the minimum angle of resolution, IOFB intraocular foreign body

${ }^{a} \mathrm{VA}$ at final follow-up could not be determined in 4 patients of non-endophthalmitis group

inoculated microorganisms [16-21]. Among these, the recognition of high-risk injury settings is necessary to effectively facilitate a specific prevention program. From previous reports, the situations related to post-traumatic endophthalmitis were varied (e.g. mostly associated with trauma caused by needles or projectile metallic foreign bodies by Faghihi et al., industrial tool use by Yang et al., and digging during farm work and hammering on metal by Asencio et al.) $[15,18,20]$. In this study, the significant etiologies of OGI, either with or without accompanied endophthalmitis, were from activities producing highvelocity objects (lawn mowing, chiseling, and hammering). Therefore, while performing those at-risk activities, eye safety measures should be applied to successfully reduce the OGI and endophthalmitis incidence.

Table 6 Microbiological distribution of culture-positive posttraumatic endophthalmitis patients $(N=25)$

\begin{tabular}{ll}
\hline Microbiological proven & Number \\
\hline Gram positive (N=18) & 7 \\
Bacillus species & 4 \\
Staphylococcus coagulase negative & 3 \\
Streptococcus pneumoniae & 2 \\
Staphylococcus epidermidis & 1 \\
Streptococcus viridan & 1 \\
Staphylococcus aureus & \\
Gram negative (N=3) & 1 \\
Enterobacter cloacae & 1 \\
Serratia marcescens & 1 \\
Escherichia coli & \\
Mixed organism (N=3) & 1 \\
Streptococcus pneumoniae/Bacillus species & 1 \\
Enterococcus faecalis/Pantoea agglomerans & 1 \\
Escherichia coli Enterobacter cloacae & \\
Fungus (N=1) & \\
Paecilomyces species & 1 \\
\hline
\end{tabular}

The relationship between the presence of IOFB and occurrence of endophthalmitis following OGI remains controversial [18-22]. Some studies confirmed an association between IOFB characteristics and the occurrence of endophthalmitis: higher risk in a wooden IOFB; and lower risk in a metallic foreign body with high velocity and high temperature as from explosion [23, 24]. However, Essex et al. indicated overall filthiness of OGI, rather than the presence of IOFB, was a risk of subsequent infection [17]. The inconclusive results may be attributed to differences in foreign body properties such as composition (metal or non-metal), contamination (clean or dirty), as well as the size of the causative material. In this study, a significant association between IOFB and endophthalmitis development was established as most IOFBs in this study were from lawn mowing-related gardening work, which was likely to be contaminated. Further evaluation in prospective studies may require elucidating a more evident relationship.

Apart from the IOFB, the time interval from injury to hospital and primary management were associated with the infection, consistent with other studies [18-20]. A large number of patients in this study presented to the hospital after $24 \mathrm{~h}$ following injury. The delayed presentation mostly related to an unawareness of the injuryrelated risks (until the development of infectious symptoms) by patients. This could be partly due to a high proportion of patients with self-sealed wounds which could appear nearly normal. Consequently, the delayed presentation allowed the amplification of microorganisms inside the eyeball. In this study, patients with selfsealing wounds had a greater number of IOFB, delayed presentation, and endophthalmitis. Therefore, patients with eye injuries, particularly those sustaining trauma from high-velocity objects, should receive prompt ophthalmic examinations with high concerns regarding the risk of intraocular infection, even when presenting with negligible symptoms. 
Table 7 Presentations and outcomes of open globe injury divided into patients with primary self-sealing wound and patients requiring primary wound repair

\begin{tabular}{|c|c|c|c|}
\hline \multirow[t]{2}{*}{ Characteristics } & \multicolumn{2}{|c|}{ Open globe injury $(N=591)$} & \multirow[t]{2}{*}{$P$ value } \\
\hline & $\begin{array}{l}\text { Self-sealing } \\
\text { wound }(N=137)\end{array}$ & $\begin{array}{l}\text { Non-self-sealing } \\
\text { wound }(N=454)\end{array}$ & \\
\hline Workplace based injury, n (\%) & $116(84.7)$ & $259(57.0)$ & $<0.001$ \\
\hline Projectile-related objects (mowing and chiseling), n (\%) & $76(55.5)$ & $144(31.8)$ & $<0.001$ \\
\hline Zone I injury, n (\%) & $92(67.2)$ & $189(41.6)$ & $<0.001$ \\
\hline Presence of IOFB, n (\%) & $100(73.0)$ & $94(20.7)$ & $<0.001$ \\
\hline Presence of endophthalmitis, $n(\%)$ & $52(38.0)$ & $66(14.5)$ & $<0.001$ \\
\hline Lens capsule rupture, n (\%) & $17(12.4)$ & $87(19.2)$ & 0.091 \\
\hline Presenting to hospital > 24 h, n (\%) & $114(83.2)$ & $170(37.4)$ & $<0.001$ \\
\hline Mean initial (SD) VA, logMAR & $1.8(0.9)$ & $2.4(0.6)$ & $<0.001$ \\
\hline Final VA worsen than $20 / 400, n(\%)^{a}$ & $56(40.9)$ & $226(50.2)$ & 0.088 \\
\hline
\end{tabular}

$V A$ visual acuity, $S D$ standard deviation, logMAR logarithm of the minimum angle of resolution, IOFB intraocular foreign body

${ }^{a} \mathrm{VA}$ at final follow-up could not be determined in 4 patients of non-endophthalmitis group

Regarding wound location, some authors reported that OGI patients with corneal laceration had a higher risk of infection $[18,25]$. Similarly, this study found that anterior wound location was a risk for occurrence of endophthalmitis. It is assumed that the more posterior wound location, particularly with intact conjunctiva, decreased the chance of the external pathogen inoculations.

To prevent endophthalmitis, an appropriate and timely antibiotic treatment is one of the essential strategies. Several methods of antibiotic administrations have been investigated in previous publications. Among these, effective managements in reducing the risk of posttraumatic infection include an immediate initiation of empirical prophylactic systemic antibiotics (either intravenous or oral administration) at initial presentation for all OGI and an injection of intravitreal antibiotics at the time of primary repair for high-risk patients [26-29]. According to our findings, signs of endophthalmitis development during treatments and complications following intravitreal antibiotics injections were not observed, thus supporting the aforementioned managements.

In post-traumatic endophthalmitis, a wide range of culture-positive rates ( $24 \%$ to $80 \%$ ) have been published $[10,19,20,22,30]$. These variations may be attributable to differences in potential risks of infection between studies including setting and nature of injury, the use of prophylactic antibiotics, and the number of microorganisms and their susceptibility to antibiotics. A low positive culture rate $(21 \%)$ in this study might be related to a low microbial load of infected agents. In addition, the prior treatment with antibiotics before presentation to this hospital and the absence of information from vitrectomy cassette fluid may partially contribute to a low detection rate.

Visual outcomes following post-traumatic endophthalmitis is another concerning issue [10, 20, 31, 32]. This study showed a similar trend of poorer visual prognosis of patients in the endophthalmitis group than the non-endophthalmitis group. In previous reports, initial VA, IOFB, retinal detachment, and microorganism virulence were the significant factors for visual outcomes in post-traumatic endophthalmitis patients. However, this study identified that the presence of RAPD and positive culture were prognostic factors for poor final VA, which may be due to their implications of the severity of ocular tissue destruction and the large number of infectious microorganisms responsible for endophthalmitis.

Limitations of this study were a retrospective design with insufficient information regarding injury settings in particular aspects. Inclusion of clinically diagnosed endophthalmitis patients, with negative cultures, may confound the results. However, all cases with intraocular infection in previous publications may not yield the positive cultures as well. Moreover, due to the detrimental consequences of endophthalmitis and the hazard of under-detection, the clinically diagnosed cases were more acceptable than the culture-proven cases in this study and other relevant studies [10,17, 19]. The results add important information regarding risk factors and outcomes of post-traumatic endophthalmitis.

\section{Conclusion}

This study shows that OGIs with corneal laceration, presence of IOFB, or delayed primary presentation were at high susceptibility for post-traumatic endophthalmitis. A poor visual prognosis for patients with OGI with accompanying endophthalmitis challenges the physician to provide timely and proper treatment. Therefore, an immediate institution of prophylactic systemic antibiotics for suspicious OGI patients and intravitreal antibiotics for high-risk groups is recommended. 


\section{Abbreviations}

BETT: The Birmingham Eye Trauma Terminology System; Cl: Confidence interval; IOFB: Intraocular foreign body; IQR: Interquartile range; logMAR: Logarithm of the minimum angle of resolution; NPL: No perception of light; OR: Odds ratio; OGl: Open globe injury; PPV: Pars plana vitrectomy; RAPD: Relative afferent pupillary defect; SD: Standard deviation; VA: Visual acuity

\section{Acknowledgements}

We thank Barbara Metzler, a director of the Chiang Mai University English Language Team, Chiang Mai University, for help with manuscript editing.

\section{Authors' contributions}

NW, JC, VC, PK, and DP designed and conducted the study. JC, CP, SC, PW, $\mathrm{PP}$, and PS analysed the data and prepared the manuscript and figures. All authors reviewed the manuscript. The author(s) read and approved the final manuscript.

\section{Funding}

This study received funding support from the Faculty of Medicine Endowment Fund, Chiang Mai University, Thailand.

\section{Availability of data and materials}

The datasets used and/or analyzed during the current study are available from the corresponding author on reasonable request.

\section{Declarations}

\section{Ethics approval and consent to participate}

This observational study was conducted in accordance with the tenets of the Declaration of Helsinki. The protocol was approved by the Research Ethics Committee, Faculty of Medicine, Chiang Mai University, Thailand. Due to anonymized data, informed consent was exempted.

\section{Consent for publication}

Not applicable.

\section{Competing interests}

The authors declare that they have no competing interests.

\section{Author details}

'Department of Ophthalmology, Faculty of Medicine, Chiang Mai University, 110 Intavaroros Road, Maung, Chiang Mai 50200, Thailand. ${ }^{2}$ Clinical Epidemiology and Clinical Statistics Center, Faculty of Medicine, Chiang Mai University, Chiang Mai, Thailand. ${ }^{3}$ Department of Family Medicine, Faculty of Medicine, Chiang Mai University, Chiang Mai, Thailand. ${ }^{4}$ Musculoskeletal Science and Translational Research (MSTR), Chiang Mai University, Chiang Mai, Thailand.

\section{Received: 19 April 2021 Accepted: 5 July 2021}

\section{Published online: 02 August 2021}

\section{References}

1. Fabian ID, Eliashiv S, Moisseiev J, Tryfonides C, Alhalel A (2014) Prognostic factors and visual outcomes of ruptured and lacerated globe injuries. Eur J Ophthalmol 24(2):273-278

2. Kong GY, Henderson RH, Sandhu SS, Essex RW, Allen PJ, Campbell WG (2015) Wound-related complications and clinical outcomes following open globe injury repair. Clin Exp Ophthalmol 43(6):508-513

3. Li EY, Chan TC, Liu AT, Yuen HK (2017) Epidemiology of open-globe injuries in Hong Kong. Asia Pac J Ophthalmol 6(1):54-58

4. Fujikawa A, Mohamed YH, Kinoshita H, Matsumoto M, Uematsu M, Tsuiki E, Suzuma K, Kitaoka T (2018) Visual outcomes and prognostic factors in openglobe injuries. BMC Ophthalmol 18(1):138

5. Court JH, Lu LM, Wang N, McGhee CNJ (2019) Visual and ocular morbidity in severe open-globe injuries presenting to a regional eye centre in New Zealand. Clin Exp Ophthalmol 47(4):469-477

6. Guven S, Durukan AH, Erdurman C, Kucukevcilioglu M (2019) Prognostic factors for open-globe injuries: variables for poor visual outcome. Eye 33(3): 392-397
7. Okamoto Y, Morikawa S, Okamoto F, Inomoto N, Ishikawa H, Ueda T, Sakamoto T, Sugitani K, Oshika T (2019) Clinical characteristics and outcomes of open globe injuries in Japan. Jpn J Ophthalmol 63(1):109-118

8. Ahmed Y, Schimel AM, Pathengay A, Colyer MH, Flynn HW (2012) Endophthalmitis following open-globe injuries. Eye 26(2):212-217

9. Gokce G, Sobaci G, Ozgonul C (2015) Post-traumatic endophthalmitis: a mini-review. Semin Ophthalmol 30(5-6):470-474

10. Nakayama LF, Bergamo VC, de Moraes NSB (2019) Six-year epidemiological analysis of post traumatic endophthalmitis in a Brazilian hospital. Int J Retina Vitreous 5:43

11. Kuhn F, Morris R, Witherspoon CD, Mester V (2004) The Birmingham eye trauma terminology system (BETT). J Fr Ophtalmol 27(2):206-210

12 Pieramici DJ, Sternberg P, Aaberg TM, Bridges WZ, Capone A, Cardillo JA, de Juan E, Kuhn F, Meredith TA, Mieler WF, Olsen TW, Rubsamen P, Stout T (1997) A system for classifying mechanical injuries of the eye (globe). The Ocular Trauma Classification Group. Am J Ophthalmol 123(6):820-831

13. Krause L, Bechrakis NE, Heimann H, Kildal D, Foerster MH (2009) Incidence and outcome of endophthalmitis over a 13-year period. Can J Ophthalmol 44(1):88-94

14. Sharma S, Padhi TR, Basu S, Kar S, Roy A, Das T (2014) Endophthalmitis patients seen in a tertiary eye care centre in Odisha: a clinicomicrobiological analysis. Indian J Med Res 139(1):91-98

15. Yang XB, Liu YY, Huang ZX, Mao Y, Zhao L, Xu ZP (2018) Clinical analysis of 1593 patients with infectious endophthalmitis: a 12-year study at a tertiary referral center in Western China. Chin Med J 131(14):1658-1665

16 Thompson WS, Rubsamen PE, Flynn HW, Schiffman J, Cousins SW (1995) Endophthalmitis after penetrating trauma. Risk factors and visual acuity outcomes. Ophthalmology 102(11):1696-1701

17. Essex RW, Yi Q, Charles PG, Allen PJ (2004) Post-traumatic endophthalmitis. Ophthalmology 111(11):2015-2022

18. Faghihi H, Hajizadeh F, Esfahani MR, Rasoulinejad SA, Lashay A, Mirshahi A, Karkhaneh R, Tabatabaey A, Khabazkhoob M, Faghihi S (2012) Posttraumatic endophthalmitis: report no. 2. Retina 32(1):146-151

19. Dehghani AR, Rezaei L, Salam H, Mohammadi Z, Mahboubi M (2014) Post traumatic endophthalmitis: incidence and risk factors. Glob J Health Sci 6(6):68-72

20. Asencio MA, Huertas M, Carranza R, Tenias JM, Celis J, Gonzalez-del Valle F (2016) A case-control study of post-traumatic endophthalmitis at a Spanish hospital. Int Ophthalmol 36(2):185-194

21. Rishi E, Rishi P, Koundanya W, Sahu C, Roy R, Bhende PS (2016) Posttraumatic endophthalmitis in 143 eyes of children and adolescents from India. Eye 30(4):615-620

22. Andreoli CM, Andreoli MT, Kloek CE, Ahuero AE, Vavvas D, Durand ML (2009) Low rate of endophthalmitis in a large series of open globe injuries. Am J Ophthalmol 147(4):601.e2-608.e2

23. Jonas JB, Knorr HL, Budde WM (2000) Prognostic factors in ocular injuries caused by intraocular or retrobulbar foreign bodies. Ophthalmology 107(5): 823-828

24. Erdurman FC, Hurmeric V, Gokce G, Durukan AH, Sobaci G, Altinsoy HI (2011) Ocular injuries from improvised explosive devices. Eye 25(11): 1491-1498

25. Schmidseder E, Miño de Kaspar H, Klauss V, Kampik A (1998) Post-traumatic endophthalmitis after penetrating eye injuries. Risk factors, microbiological diagnosis and functional outcome. Ophthalmologe 95(3):153-157

26. Narang S, Gupta V, Gupta A, Dogra MR, Pandav SS, Das S (2003) Role of prophylactic intravitreal antibiotics in open globe injuries. Indian J Ophthalmol 51(1):39-44

27. Tabatabaei SA, Soleimani M, Behrooz MJ, Sheibani K (2016) Systemic oral antibiotics as a prophylactic measure to prevent endophthalmitis in patients with open globe injuries in comparison with intravenous antibiotics. Retina 36(2):360-365

28. Du Toit N, Mustak S, Cook C (2017) Randomised controlled trial of prophylactic antibiotic treatment for the prevention of endophthalmitis after open globe injury at Groote Schuur Hospital. Br J Ophthalmol 101(7): 862-867

29. Abouammoh MA, Al-Mousa A, Gogandi M, Al-Mezaine H, Osman E, Alsharidah AM, Al-Kharashi A, Abu El-Asrar AM (2018) Prophylactic intravitreal antibiotics reduce the risk of post-traumatic endophthalmitis after repair of open globe injuries. Acta Ophthalmol 96(3):e361-e365

30. Vedantham V, Nirmalan PK, Ramasamy K, Prakash K, Namperumalsamy P (2006) Clinico-microbiological profile and visual outcomes of post-traumatic 
endophthalmitis at a tertiary eye care center in South India. Indian J Ophthalmol 54(1):5-10

31. Gupta A, Srinivasan R, Gulnar D, Sankar K, Mahalakshmi T (2007) Risk factors for post-traumatic endophthalmitis in patients with positive intraocular cultures. Eur J Ophthalmol 17(4):642-647

32. Cornut PL, Youssef EB, Bron A, Thuret G, Gain P, Burillon C, Romanet JP, Vandenesch F, Maurin M, Creuzot-Garcher C, Chiquet C, French Institutional Endophthalmitis Study (FRIENDS) Group (2013) A multicentre prospective study of post-traumatic endophthalmitis. Acta Ophthalmol 91(5):475-482

\section{Publisher's Note}

Springer Nature remains neutral with regard to jurisdictional claims in published maps and institutional affiliations.

Submit your manuscript to a SpringerOpen ${ }^{\mathcal{O}}$ journal and benefit from:

- Convenient online submission

- Rigorous peer review

- Open access: articles freely available online

High visibility within the field

- Retaining the copyright to your article

Submit your next manuscript at $\boldsymbol{\nabla}$ springeropen.com 